\title{
INTEROPERABILIDADE SEMÂNTICA E ONTOLOGIA SEMIÓTICA: A CONSTRUÇÃO E O COMPARTILHAMENTO DE CONCEITOS CIENTÍFICOS EM AMBIENTES COLABORATIVOS ONLINE
}

\section{LA INTEROPERABILIDAD SEMÁNTICA Y ONTOLOGÍA SEMIÓTICA: LA CONSTRUCCIÓN Y ADOPCIÓN EN COMÚN DE LOS CONCEPTOS CIENTÍFICOS EN LA COLABORACIÓN EN LÍNEA}

\author{
Maria Aparecida Moura - cidamoura@gmail.com \\ Professora Associada da Escola Ciência da \\ Informação da UFMG. Doutora em Comunicação e \\ Semiótica pela PUC/SP.
}

\begin{abstract}
Resumo
A consolidação dos sistemas de informação cooperativos, intensificados pela Web 2.0 e a institucionalização progressiva do conceito de E-Science no contexto da pesquisa científica, aumentou significativamente a presença de pesquisadores no ambiente digital e criou novas dinâmicas de consolidação colaborativa de conceitos científicos. Neste trabalho, tomaram-se por referência as principais evidências da dinâmica de construção colaborativa de conceitos científicos presentes nos discursos e na comunicação científica contemporânea na WEB para produzir um experimento de interoperabilidade semântica por meio de uma ontologia semiótica. $\mathrm{O}$ corpus terminológico foi constituído a partir de uma amostra de blogs científicos mantidos por pesquisadores como estratégia para o registro e a divulgação dos resultados parciais de pesquisa, sites de colaboratórios internacionais e de centros internacionais que apoiam as práticas EScience e as chamadas de trabalho em eventos científicos vinculados à colaboração, à inovação científica e à pesquisa em ambientes digitais no período de 2007 a 2010. Como resultado, consolidou-se uma ontologia semiótica composta pela estrutura semântica pactuada pelos pesquisadores em ambientes digitais demarcados pelo trabalho colaborativo e pelo compartilhamento conceitual em rede.
\end{abstract}

\section{Palavras-chave}

Interoperabilidade semântica. Ontologia semiótica. Redes de cooperação científica.

\section{INTRODUÇÃO}

"A terminologia é o momento poético do pensamento" (Giorgio Agamben) 
Nos últimos anos, o fortalecimento da concepção do conhecimento como linguagem exigiu dos teóricos da organização da informação grande investimento intelectual em direção às teorias da significação como possibilidade de ampliação da compreensão e fortalecimento da representação temática em sistemas de informação.

Nesse período, ampliou-se a penetração e o apelo às epistemologias sem sujeito cuja força explicativa se localiza prioritariamente no signo e nos processos de significação. Todavia, o lugar do sujeito na teoria semiótica é ocupado por uma mente interpretadora. Assim, se o período priorizou fundamentalmente o signo, a sua atribuição requer a participação ativa de um sujeito intérprete.

Há muitos anos os pesquisadores do campo da organização de informação e do conhecimento desenvolvem pesquisas e experimentos que visam valorizar a linguagem natural com estratégia de organização e recuperação da informação. Os esforços de consolidação de métodos modernos remetem a Taube em 1951, quando este propôs o sistema UNITERM. Esse modelo teve muita repercussão e influência no desenvolvimento de sistemas de recuperação de informação a partir dos anos 50.

Passados 60 anos de pesquisas, o uso de linguagens verbais em sistemas de organização e recuperação da informação ainda é um desafio central nos estudos desenvolvidos no campo da Ciência da Informação e em suas áreas fronteiriças.

Além disso, a popularização do acesso e a expansão da digitalização em todos os domínios da vida permitiu uma incontestável sobreposição entre as esferas de produção e utilização da informação e ampliou exponencialmente o surgimento de produtos e serviços voltados à organização da informação em ambientes colaborativos.

Hoje, vivemos sob a égide da produção da informaçãosob demanda, dos mecanismos colaborativos online e submetidos aos novos padrões de arbitragem do conhecimento o que têm ocasionado radicais transformações na produção e disseminação da informação e, consequentemente, exigem alterações nas metodologias de elaboração dos instrumentosverbais de representação da informação. Muitas iniciativas foram tomadas, mas ainda se verifica um desequilíbrio nas articulações teóricas e nos consequentes experimentos. Ora privilegia-se o tecnológico, ora privilegia-se a experimentação decorrente da experiência e em outros momentos reitera-se a distinção teórica envolvida na questão. (MOURA, 2009, p. 60) 
Diante disso, os processos de elaboração de dispositivos voltados à organização temática da informação, passaram a sinalizar a necessidade de mudanças com a possibilidade de interoperabilidade semântica entre os instrumentos. Isto se deve, sobretudo, à relativa semelhança nos processos de coleta e tratamento dos corpora terminológicos que, por vezes, se sobrepõem quando pensa-se nos ambientes colaborativos em rede.

\section{FERRAMENTAS ONTOLÓGICAS E A INTEROPERABILIDADE SEMÂNTICA}

As ferramentas ontológicas (MOURA, 2009, p. 62) são dispositivos informacionais contextualizados e desenvolvidos sobumponto de vistaespecífico com objetivo de orientar a organização da informação temática em um dado contexto informacional. Taisferramentas têm porobjetivoorientar os atores sociais no entendimentoacerca do conhecimentoemáreas específicas bemcomo na adoçãoconsciente desses esquemas representacionais emsistemas de organização e recuperação da informação. As ferramentas ontológicas têm sido utilizadas para designar os estudos conceituais específicos que visam caracterizar dada área de conhecimento a partir do mapeamento das suas categorias gerais e específicas. Entendem-se as ferramentas ontológicas como um tipo de dispositivo que, de acordo com $\operatorname{Agamben}^{1}$ (2009, p. 14) é tudo o que, de uma forma ou de outra, tem a capacidade de capturar, orientar, determinar, interceptar, modelar, controlar e assegurar os gestos, as condutas, as opiniões, ou os discursos dos seres vivos.

Nesse contexto de análise a interoperabilidade refere-se à capacidade de sistemasautônomos (informatizados ounão) comunicarem de modotransparenteentresi, devido à adoção de padrõescomuns e protocolosque permitem o uso compartilhado de informações. A interoperabilidade desenvolvida no contextodigital tomacomo referência o estabelecimento da representação estrutural, sintática, semântica e lógica.

De acordo com Degoulet et al., (2011), a interoperabilidade sintática refere-se a uma integração de primeiro nível que conduz à noção de sistema aberto e permite a incorporação da heterogeneidade dos componentes no sistema.

\footnotetext{
${ }^{1}$ I shall call an apparatus literallyanything that has in some way the capacity to captureorient, determine, intercept, model, control, or secure the gestures, behaviors, opinions,or discourses of living beings. ( AGAMBEN, 2009, p.14)
} 
A interoperabilidade semântica, considerada uma integração de segundo nível, caracteriza-se pela interconectividade e a garantia sistêmica de que as informações compartilhadas entre diferentes sistemas e aplicações poderão ter seus significados compreendidos e operacionalizados em distintas etapas do desenvolvimento de um projeto. Para garantir a compatibilidade dos dados e dos procedimentos adotados é necessário o compartilhamento de convenções e protocolos de comunicação e um sistema conceitual subjacente às diferentes aplicações.

Degoulet et al., (2011) salientam que a interoperabilidade semântica requer a articulação de três elementos: a terminologia, a explicitação das relações semânticas dos elementos da terminologia e a representação dos conceitos nas mensagens.

A terminologia se refere ao vocabulário controlado relacionado a um domínio de conhecimento ou atividade. As relações semânticas articulam a rede de conceitos do domínio e expressam a natureza de cada conceito que a integra. A representação dos conceitos nas mensagens visa compatibilizar as diferentes formas através das quais as organizações se referem aos dados semelhantes.

De acordo com Dahlberg (1978) o conceito caracteriza-se pela reunião e a compilação de enunciados verdadeiros a respeito de determinado objeto.

Mellal (2007) mapeou algumas estratégias de interoperabilidade semântica em ontologias. De acordo com a autora as estratégias são o mapeamento, a fusão e o alinhamento de ontologias.

O mapeamento visa explicitar as correspondências semânticas entre as ontologias. A fusão permite criar uma nova ontologia a partir da incorporação do conhecimento de outras ontologias. A estruturação de novas ontologias pela fusão tem como principal desafio a garantia de correspondência semântica entre as matrizes e o instrumento derivado. $\mathrm{O}$ alinhamento tem por objetivo descobrir correspondências entre as ontologias em negociação permitindo estabelecer vínculos semânticos entre os conceitos e as relações. 
Figura 1 - Mapeamento entre duas ontologias locais

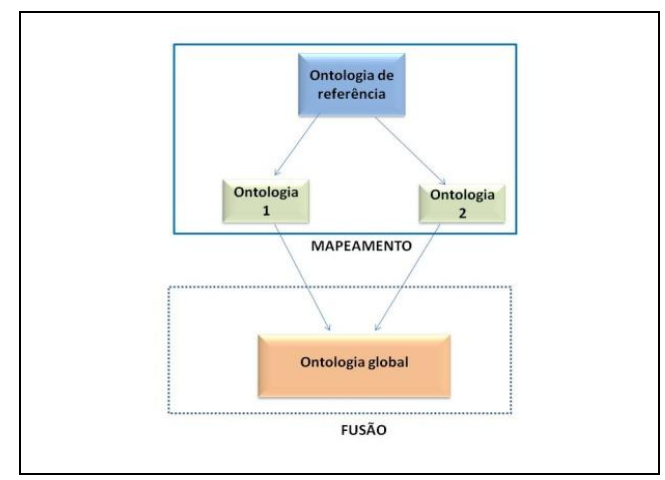

Fonte: Mellal; Naçima (2007).

O mapeamento de ontologias pode ser feito com o apoio de softwares, tais como o MApping Framework (MAFRA) voltado ao mapeamento em sistemas distribuídos.

\section{A ONTOLOGIA SEMIÓTICA COMO SISTEMA CONCEITUAL HÍBRIDO}

Na década de 90 do século XX Lancaster (1993) defendia a necessidade de criação de um sistema conceitual híbrido que compreendesse a complexidade da organização da informação perpassada pelo imperativo tecnológico. Segundo o autor,

O termo híbrido é empregado para designar qualquer sistema que funcione com uma combinação de termos controlados e linguagem natural, inclusive aqueles em que ambos os conjuntos de termos são atribuídos por indexadores humanos e aqueles em que uma base de dados pode ser consultada mediante uma combinação de termos controlados atribuídos por seres humanos e palavras que ocorram nos títulos, resumos ou texto completo. (1993, p. 223).

Em vista disso, o principal desafio hoje é criar metodologias que permitam combinar a dinâmica da atribuição livre com o rigor e a complexidade do controle de vocabulário nos processo de representação e recuperação da informação.

Diante desse conjunto de complexidades, característico dos processos de organização da informação, teve inicio no ano de 2007 a implementação do projeto ISO 25964-1:2011 desenvolvido pela NISO que visa estabelecer a interoperabilidade entre tesauros e outros vocabulários adotados como esquemas de representação da informação. Na primeira parte do projeto, publicada em agosto de 2011, foram analisados os tesauros monolíngues e multilíngues e sua função na recuperação da informação. Em 2012, está previsto a estruturação da norma técnica que terá como base a interoperabilidade entre as interfaces conceituais (taxonomias, ontologias, dentre outros). 
A medida é amplamente aguardada pelos profissionais que se dedicam ao desenvolvimento das referidas ferramentas.

O foco da proposta é a padronização sintática e semântica das relações entre os conceitos com vistas a promover a compatibilidade entre os sistemas de organização da informação e do conhecimento. Na etapa da interoperabilidade propriamente dita pretende-se focalizar o mapeamento das relações entre os conceitos em um vocabulário específico e entre vocabulários distintos. De acordo com os propositores da norma as relações centrais continuarão sendo fundamentalmente a equivalência, a hierarquia e as relações associativas. Todavia, acredita-se que em função das múltiplas possibilidades de formalização das relações ontológicas, notadamente as características acidentais dos conceitos, será possível fornecer expressividade às relações funcionais e apoiar a sedimentação das definições nominais pautadas pelo uso social dos conceitos.

Devido às características tecnológicas e estruturais das ontologias acredita-se que o esforço de integração das ferramentas ontológicas ora empreendida pela NISO poderá ocorrer incorporando o elemento semiótico em sua estruturação.

De acordo com Almeida (2006, p. 106) as ontologias podem ser consideradas tanto um objeto, quanto um processo. Para o autor, as ontologias viabilizam o estudo de "uma série de formalismos capazes de representar os conceitos, as relações entre os conceitos e a semântica de um domínio do conhecimento".

Segundo (MELLAL, 2007, p. 56), as ontologias apresentam como vantagens a possibilidade de reuso, a preservação semântica dos conhecimentos e a possibilidade de interoperabilidade semântica. Elas se dividem em: ontologias estáticas que descrevem entidades existentes em um contexto de atributos e relações; ontologias dinâmicas que incorporam os aspectos dinâmicos dos contextos sociais e possuem foco nos estados e processos; ontologias intencionais, que expressam tarefas, objetivos e crenças de agentes situados em um determinado ambiente e as ontologias sociais que cobrem os aspectos sociais.

De acordo Zacklad (2005, p. 4) as ontologias semióticas são produções semióticas coerentes que reagrupam expressões extraídas de transações comunicacionais e são organizadas segundo eixos paradigmáticos e sintagmáticos. As expressões selecionadas são consideradas como conceitos oriundos de um processo de investimento em definições. 
Nesse sentido, podem ser consideradas ontologias de domínio que se baseiam na incorporação de elementos semióticos traduzidos por dispositivos semânticos que auxiliam na explicitação aproximada e compreensão dos processos interpretativos de atores sociais em situações de organização, disseminação e recuperação de informações em rede. A viabilização das ontologias semióticas permite, a partir de uma base terminológica estruturada, explicitar e formalizar os pontos de vista envolvidos nas ações relacionadas à comunicação da informação (instância gerativa), representação da informação (instância de intermediação) e o contexto interpretativo (recepção). Tomam como referência o diálogo, a co-construção [estou sem o dicionário novo, mas me parece que é correto sem hífem] permanente no âmbito das comunidades de referência do domínio (identificação, sistematização, validação e apropriação coletiva) bem como a explicitação dos pontos de vista das comunidades de saberes implicadas na operacionalização dos fluxos e circulação da informação.

De acordo do Zacklad (2005, p. 3),

Devido ao fato da distribuição das atividades coletivas, realizadores e beneficiários de transações comunicacionais podem não estar presentes em um mesmo quadro espaço-temporal. Isso implica em estruturar estratégias que permitam prolongar estas transações de maneira que elas possam ser iniciadas, interrompidas, atualizadas, repetidas, em todas as configurações de presença ausente do beneficiário ou realizador. Nós definimos assim diversas estratégias de distribuição espaço-sócio-temporal de transações: a normalização da situação transacional, a formalização da expressão, a ritualização mnemotécnica, a abstração, a mediação substitutiva e a documentação.

Assim, a estruturação de conceitos na ontologia pode ser determinada, todavia a sua integração no dispositivo deve ser articulada com base em um acordo de definição conceitual explicitado e controlado por uma comunidade de referência.

De acordo com Zacklad (2005) as abordagens adotadas na configuração da ontologia semiótica são lógicas, contextuais e situacionais.

A abordagem lógica, fundamento das ontologias formais, baseia-se na significação independente do contexto semiótico da expressão e independente da situação de comunicação transacional.

A abordagem contextual toma como referência a produção semiótica em sua totalidade. Nessa abordagem a importância da sintaxe é relativizada e são enfatizadas as expressões do gênero e da proximidade de uma zona de significação específica no documento. 
A abordagem situacional evidencia uma prática social contínua e focaliza a significação na situação transacional. Nesse caso, a situação transacional é pautada por projetos comuns, a natureza das relações sociais entre os participantes, as características do quadro espaço-temporal e as condições ambientais e o compartilhamento de um campo representacional comum. Nesta abordagem o investimento ontológico requer um acordo entre os participantes em relação ao conteúdo semiótico das expressões.

Nesse contexto, os acordos de definição pautam-se pela existência de um mundo objetivo. Para a composição das definições tomam como referência uma abordagem funcional que se orienta pela situação transacional.

Para Zacklad (2005, p. 7) a formalidade semiótica pode reduzir a padronização das situações transacionais de referência e permitir a abertura do sentido multiplicando as ancoragens possíveis de um conceito a partir de diferentes pontos de vista. Esse parece ser um paradoxo importante, se considerarmos que a formalidade tecnológica busca a redução do deslizamento do significado e a perenidade do sentido. Todavia, a univocidade do signo já não se coaduna com as necessidades e os perfis informacionais contemporâneos.

\section{A CONSTRUÇÃO DE BASES TERMINOLÓGICAS MULTIUSUÁRIOS}

O projeto "Informação, Gêneros Digitais e Discurso Científico: desafios e estratégias na modelagem, organização e disseminação da informação em ambientes colaborativos", têm por objetivo identificar, caracterizar e modelar os processos de interação e colaboração entre os atores sociais na produção de informação em redes científicas. Adotaram-se como mediação as pistas encontradas na linguagem, nos discursos e nos sistemas teóricos e metodológicos compartilhados em web gêneros disponíveis em repositórios de informação digital de acesso aberto. Buscou-se compreender as implicações dos acordos e da interpenetração semiótica do discurso científico contemporâneo nos sistemas de organização da informação e do conhecimento.

Para tanto, analisaram-se os gêneros digitais (webgenre ou cybergenre) utilizados pelos pesquisadores em situações de compartilhamentos de conhecimento. Os gêneros digitais referem-se às categorias documentais emergentes em termos estruturais e discursivos que possuem traços derivados do caráter multimodal e hipertextual consolidado no contexto da web nos últimos anos. Os gêneros digitais têm se expandido 
no contexto científico em virtude de sua multifuncionalidade no que concerne a produção, circulação e recepção da informação.

Devido ao investimento na interoperabilidade entre os dispositivos verbais dedicados à representação da informação tornaram-se necessários novos dispositivos, abordagens e fontes para a identificação e a consolidação da terminologia a ser utilizada nos vocabulários. Acredita-se que é urgente incorporar as ferramentas disponíveis nos ambientes digitais no processo de criação dos referidos vocabulários. Nesse sentido, ao estudar os ambientes colaborativos científicos buscou-se compreender com se constroem cooperativamente os consensos em torno dos conceitos em ambientes digitais.

No estudo desenvolvido tomaram-se por referência as principais evidências da dinâmica de construção colaborativa de conceitos científicos presentes nos discursos e na comunicação científica contemporânea na WEB para produzir um experimento de interoperabilidade semântica. O corpus terminológico foi constituído a partir de uma amostra de blogs científicos mantidos por pesquisadores como estratégia para o registro e a divulgação dos resultados parciais da pesquisa, sites de colaboratórios internacionais e de centros internacionais que apoiam as práticas E- Science e as chamadas de trabalho em eventos científicos vinculados à colaboração, a inovação científica e à pesquisa em ambientes digitais no período de 2007 a 2010.

Para a seleção dos blogs científicos analisados tomou-se por referência os indicadores descritos por Efimova (2009, p. 40), a saber, a autenticidade (representado pelo estudo de um fenômeno social), confiabilidade (qualidade da pesquisa), impacto (medido pelo engajamento dos leitores, as interações entre o pesquisador e seus leitores e à transmissão de mensagens no blog com comentários sobre a pesquisa atual).

Os colaboratórios identificados na pesquisa referem-se a estruturas de comunicação e serviços voltados à colaboração científica que se realizam em torno do compartilhamento de computação distribuída, informações digitais retrospectivas e prospectivas e de interfaces digitais amigáveis (ubíquas, multimodais, nômades e imersivas).

$\mathrm{Na}$ etapa de identificação dos colaboratórios elaborou-se uma base de dados na qual se buscou sistematizar as concepções de colaboração e os sistemas conceituais pactuados em tais ambientes. 
$\mathrm{Na}$ Ontology of Semantics, Knowlegde and Gridque resultou da referida pesquisa, partiu-se de um conjunto de conceitos representativos do contexto estudado para operar a coleta de novos conceitos em contexto.

Em seguida, foi criado o Online Dictionary of E-Science, Cyberculture and Scientific Narratives com o propósito de, através da formalização dos sistemas conceituais pactuados, compreender os elementos lógicos, contextuais e situacionais presentes na linguagem adotada.

Figura 2 - Interface do dicionário proposto

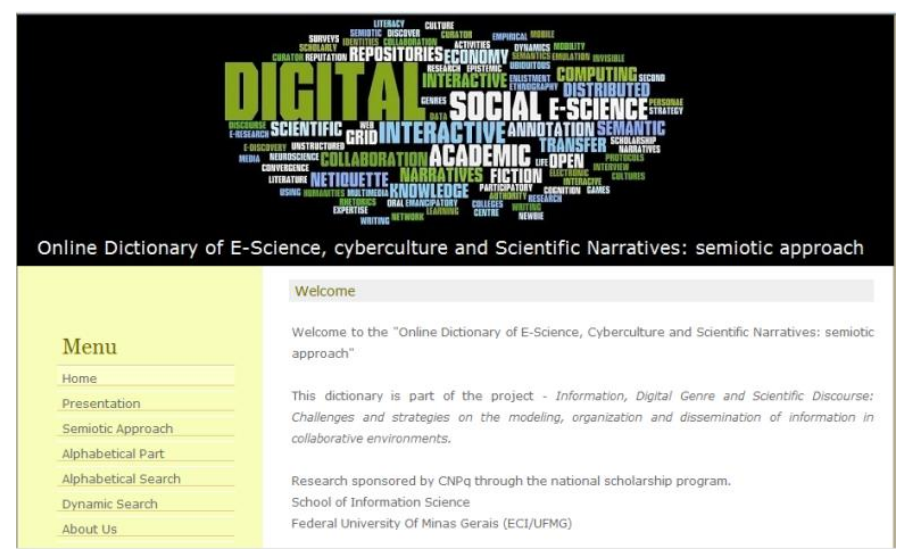

\section{Fonte: Da autoria}

$\mathrm{Na}$ etapa posterior, foram consultados os termos em contexto com a ajuda do software webcorp ${ }^{2}$. Nessa etapa foi possível apreender a dinâmica dos acordos de definição e o compartilhamento colaborativo de conceitos científicos empreendidos pelos pesquisadores em ambientes digitais. Em seguida estabeleceram-se as categorizações dos conceitos e a sua formalização no software Ontoeditor.

A rede semântica proposta foi composta por 600 conceitos provenientes do universo colaboração científica em ambientes digitais. Os conceitos foram classificados nas seguintes categorias: estratégia e gestão de projetos; captura de dados; softwares; publicação e divulgação de dados; comunicação e colaboração; colaboração cultural, estruturação de dados; organismos de financiamento; e-infraestruturas para a pesquisa; curadoria digitais; abordagens teóricas da Informação; filosofia da tecnologia; intercâmbio de conhecimentos; serviços de publicação, padrões de desenvolvimento de dados; impacto social da inovação; trabalho interdisciplinar; organização e gestão, operações e apoio; rede social; dinâmica de colaboração e pesquisa.

${ }^{2}<$ http://www.webcorp.org.uk:8080/index.jsp> 
Em seguida, foram sistematizados os principais motivos e estratégias adotadas pelos pesquisadores para criar um novo conceito científico. As estratégias foram estruturadas de acordo com as práticas mais recorrentes na composição do conceito dentre as quais destacam-se: neologismos,conceitos estabelecidos, retrónimos, fases evolutivas do conceito e acrônimos.

A amostra da pesquisa indicou que hoje os conceitos científicos no contexto analisadosão formados por $56 \%$ de neologismos, marcados pelas palavras significativas a partir do contexto, $16 \%$ de conceitos já estabelecidos, $14 \%$ de retronimos, $8 \%$ de acrônimos e $6 \%$ referentes a indicação das fases evolutivas dos fenômenos e conceitos.

Figura 3 - Formação de conceitos científicos em ambientes digitais

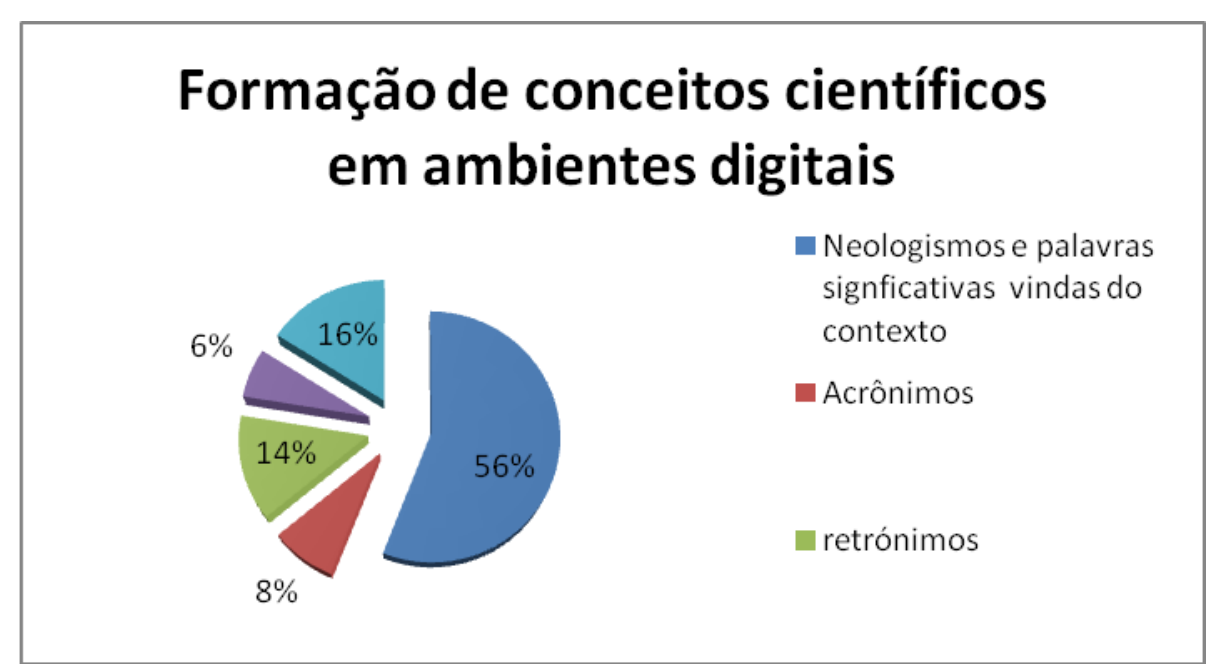

Fonte: da autoria

- Neologismos - Criação de novos conceitos através da combinação de palavras existentes ou dando novas palavras e único com os prefixos ou sufixos. Exemplo: colaboratório, cibercultura, cibersociabilidade, blogosfera, folksonomia, hashtag, hipermídia.

- Retrónimos - O primeiro conceito é diferenciado deste último para reforçar as singularidades ou inovações. Exemplo: vírus de computador, direitos autorais, copyleft, curadoria de dados, alfabetização digital.

- Siglas - O conceito é formado a partir da primeira letra de um conjunto de palavras que o compõe, exemplo: WEB, HTML, FIDE, FAQs, FOAP.

- Fases evolutivas - Como os esquemas de controle de versão de software, os novos conceitos são mencionados com um número para diferenciar ou para mostrar a 
melhoria e a ordem crescente em relação ao precedente. Exemplo: ciência 2.0, web 2.0, pesquisa 2.0, biblioteca 2.0, Identidade 2.0.

- Inclusão de palavras significativas do contexto para mostrar o novo uso, restrição ou aplicação - (cyber, web, E-, online, virtual, aberto, multi, rede, net, participativa, comum, social, auto, ubíquo, acadêmica, público, colaboração, etc.) Exemplo: cibercrime, E-pesquisa, arquivos abertos.

As reuniões científicas analisadas pertencem aos seguintes campos conhecimento: Sociologia do conhecimento, Estudos de Internet, Comunicação Científica, Ciência da Computação, Ciência da Informação, Educação, Engenharia, Design Participativo, História Conceitual e Pensamento Político, entre outros.

\section{CONSIDERAÇÕES FINAIS}

Nosso estudo nos permitiu identificar a inovação científica do trabalho colaborativo em termos de sua manifestação como linguagem. Isso foi possível, através da construção de uma rede semântica compartilhada pelos pesquisadores em reuniões científicas,colaboratórios e blogs científicos.

O estudo da história da construção e consolidação de conceitos científicos em processo nos permitiu compreendê-los à luz da experiência humana expressa através da linguagem, bem como das lutas semânticase suas implicações na construção social da realidade.

Foi possível apreender a importância da formalização semiótica nos investimentos em dispositivos ontológicos para a organização da informação na medida em que tornouse possível incorporar e modelar a movimentação sócio-espaço-temporal das transações comunicacionais em contextos científicos. De acordo com nossa experiência na construção da ontologia, a utilização dos instrumentos disponíveis na Web para o desenvolvimento de ferramentas ontológicas provou ser válido. Isso se deve a rapidez com que os recursos informacionais são disponibilizados, o que exige uma redução drástica entre a produção conhecimento, a representação e a circulação da informação dele decorrentes.

O estudo evidenciou que as ferramentas para a representação temática da informação ainda pemanecem como bons instrumentos de mediação da informação no 
contexto digital devido a perenidade do conjunto de normas adotadas em seu estabelecimento, haja vista o esforço internacional em curso voltado à interoperabilidade dos vocabulários.

A triangulação de métodos de abordagem de bases terminológicas experimentada mostrou-se promissora, sobretudo em contextos de intensa negociação semântica como aquele analisado.

A análise dos resultados demonstrou que as três instâncias usadas para investigar a criação de novos conceitos são apropriadas, porque referem-se a espaços virtuais voltados à experimentação e a colaboração, fundamentais para criação e formalização de consensos e a estabilidade conceitual necessárias aos avanços científicos.

Além disso, como constata Zacklad (2005) os investimentos em definições conceituais em ambientes sociais online podem facilitar as traduções entre os sentidos pactuados emsituações de transação comunicacional e conversacional específicos e as significações já estabelecidas em acordos e convenções pautados pela abordagem lógica em comunidades científicas. Tal procedimento pode auxiliar na consolidação e atualização de dispositivos conceituais mais dinâmicos e adaptáveis aos contextos sociais em constante transformação.

Portanto, acredita-se que se incorporarmos criticamente as pistas advindas do discurso e das práticas das comunidades científicas em redes sociais, seremos capazes de melhorar as formas de representação e o acesso à informação em ambientes colaborativos e digitais.

\section{AGRADECIMENTOS}

Agradecimentos são devidos ao Conselho Nacional de Desenvolvimento Científico e Tecnológico ( $\mathrm{CNPq}$ ) pelo apoio concedido no desenvolvimento desse trabalho e a Adriane Legnani, bolsista de iniciação científica vinculada ao projeto.

\section{REFERÊNCIAS}

AGAMBEN, G. What is an apparatus? Stanford: Stanford University Press, 2009.

BARCELLOS, Mauricio Almeida. Um modelo baseado em ontologias para a construção da memória organizacional. Belo Horizonte: PPGCl/ECl, 2006. 
CLARKE, Stella G Dextre. ISO 25964: a standard in support of KOS interoperability. In: Isko Biennial Conference. 4-5 July. 2011. London. Disponível em: <http://www.iskouk.org/conf2011/papers/dextreclarke.pdf>. Acesso em: 25 de jul. de 2011.

DAHLBERG, Ingetraut. Teoria do conceito. Ciência da Informação, Brasília, v. 7, n. 2, 1978. p. 101-107.

DEGOULET, Patrice; FIESCHI, Marius; ATTALI, Christophe. Les enjeux de l'interopérabilité sémantique dans les systèmes d'information de santé. Disponível em: <http://www.spim.jussieu.fr/doc/pdg/lnfoSante/9-23.pdf>. Acesso em 25 de out. de 2011.

EFIMOVA, L. Passion at work: logging practices of knowledge workers. Enschede: Novay. 2009.

ONLINE DICTIONARY OF E-SCIENCE, CYBERCULTURE AND SCIENTIFIC NARRATIVES:semiotica pproach. Disponível em:<http://mamoura.eci.ufmg.br/dictionary/firstpage.php>. Acesso em: 08 de jul. de 2011.

GONZÁLEZ DE GÓMEZ, Maria Nélida.. A representação do conhecimento e o conhecimento da representação: algumas questões epistemológicas. Ci Inf. Brasília, v. 22 , n. 3 , p. 217-222, set./dez. 1993. Disponível em: <http://revista.ibict.br/index.php/ciinf/article/view/1128/777> . Acesso em: 08 de jul. 2011.

LANCASTER, F.W. A linguagem natural na recuperação da informação. In: Indexação e resumos: teoria e prática. Brasília: Briquet de Lemos livros, 1993. p. 200 - 228.

MELLAL, Naçima. Réalisation de l'interopérabilité sémantique des systèmes, basée sur les ontologies et les flux d'information. Chambery: Université de Savoie, 2007. Disponível em:<http://www.polytech.univsavoie.fr/fileadmin/polytech_autres_sites/sites/listic/Theses/ Manuscrit-Mellal-v-finale.pdf>. Acesso em: 24 de jul. de 2011.

MOURA, M. A. Informação, ferramentas ontológicas e redes sociais ad hoc: a interoperabilidade na construção de tesauros e ontologias. Informação \& Sociedade. Estudos, v. 19, 2009, p. 59-74.

POMBO, Olga. Da classificação dos seres à classificação dos saberes. 2002 Disponível em: <http://www.educ.fc.ul.pt/hyper/resources/opombo-classificacao.pdf>. Acesso em: 30 de maio de 2011.

SVENONIUS, Elaine. The intellectual foundation of information organization. Cambridge, Mass.: MIT Press, 2000.

ZACKLAD, Manuel. 2007. Classification, thésaurus, ontologies, folksonomies: comparaisons du point de vue de la recherche ouverte d'information (ROI). Disponível em:<http://archivesic.ccsd.cnrs.fr/docs/00/20/24/40/PDF/cais-acsi_zacklad_-

_avec_ref.pdf>. Acesso em: 30 de jul.2011.

ZACKLAD, Manuel. Introduction aux ontologies sémiotiques dans Le Web Socio Sémantique. In actes de la Conférence Ingénierie des Connaissances 2005, Nice. Disponível em: <http://hal.inria.fr/docs/00/06/26/30/PDF/sic_00001479.pdf>. Acesso em: 30 de jul. de 2011. 


\title{
Title
}

Semantic interoperability and semiotic ontology: the construction and sharing of scientific concepts in environments for online collaboration

\begin{abstract}
The consolidation of the information and collaborative systems intensified by the Web 2.0 and the progressive institutionalization of the E-Science concept in the context of scientific research has significantly increased the presence of researchers in the digital environment and has created new dynamic to discuss and consolidate the scientific concepts. In this paper, the main evidence of the dynamics of collaborative construction of scientific concepts present in the discourses and contemporary communication in the web were taken as reference to produce an experiment based on semantic interoperability through a semiotic ontology. The terminological corpus was constructed from a sample of scientific blogs maintained by researchers as a strategy to record and publicize the partial results of the research, websites from the international centers and collaboratories that support e-Science practices and the calls for papers from scientific events related with collaboration, scientific innovation and research in digital environments in the period of 2007 to 2010. As a result, a semiotic ontology was consolidated, consisting of the semantic structures from the agreements among researchers in digital contexts articulated by the collaborative work and conceptual sharing network.
\end{abstract}

\section{Keywords}

Semantic interoperability. Semiotic ontology. Scientific and collaborative networks.

\section{Título}

La interoperabilidad semántica y ontología semiótica: la construcción y adopción en común de los conceptos científicos en la colaboración en línea

\section{Resumen}

La consolidación de los sistemas de información y de colaboración intensificada por la Web 2.0 y la progresiva institucionalización del concepto de investigación digital en el contexto de la investigación científica ha aumentado significativamente la presencia de investigadores en el entorno digital y ha creado nuevas dinámicas para debatir y consolidar los conceptos científicos. En este trabajo, se tomaron como referencia, los signos de la dinámica de la construcción colaborativa de los conceptos científicos. Se produjo un experimento basado en la interoperabilidad semántica a través de una ontología semiótica. El corpus terminológico se construyó a partir de una muestra de blogs mantenidos por los investigadores científicos como una estrategia para dar a conocer los resultados parciales de la investigación, los sitios web de los centros internacionales y colaboratorios que apoyan las practicas de ciencia en línea y las convocatorias de los eventos científicos relacionados con la investigación de colaboración, la innovación científica y en los entornos digitales en el período 2007 a 2010. Como resultado, se consolidó una ontología compuesta por las estructuras semánticas de los acuerdos entre los investigadores en el contexto digital articulada por el trabajo de colaboración y uso compartido de red conceptual.

\section{Palabras clave}

Interoperabilidad semántica. Ontología semiótica. Redes sociocientíficas de colaboración.

\section{Recebido em: 10/11/2011}

Aceito em: 07/12/2011 